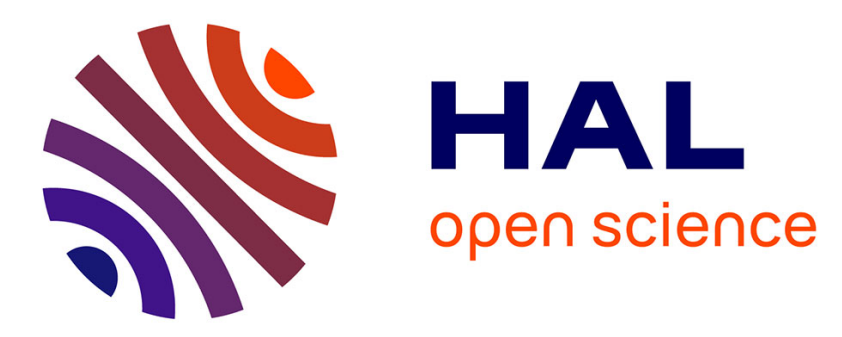

\title{
Linear macroscopic properties of polymeric liquids and melts. A new approach
}

\author{
H.R. Brand, H. Pleiner, W. Renz
}

\section{To cite this version:}

H.R. Brand, H. Pleiner, W. Renz. Linear macroscopic properties of polymeric liquids and melts. A new approach. Journal de Physique, 1990, 51 (11), pp.1065-1076. 10.1051/jphys:0199000510110106500 . jpa-00212431

\section{HAL Id: jpa-00212431 https://hal.science/jpa-00212431}

Submitted on 1 Jan 1990

HAL is a multi-disciplinary open access archive for the deposit and dissemination of scientific research documents, whether they are published or not. The documents may come from teaching and research institutions in France or abroad, or from public or private research centers.
L'archive ouverte pluridisciplinaire HAL, est destinée au dépôt et à la diffusion de documents scientifiques de niveau recherche, publiés ou non, émanant des établissements d'enseignement et de recherche français ou étrangers, des laboratoires publics ou privés. 
Classification

Physics Abstracts

$61.40 \mathrm{~K}-05.70 \mathrm{~L}-65.70$

\title{
Linear macroscopic properties of polymeric liquids and melts. A new approach
}

\author{
H. R. Brand $\left({ }^{1,2}\right)$, H. Pleiner $\left({ }^{1,3}\right)$ and W. Renz $\left({ }^{4}\right)$ \\ (1) FB 7, Physik, Universität Essen, D 4300 Essen 1, F.R.G. \\ (2) Center for Nonlinear Studies, MS-B 258, Los Alamos National Laboratory, University of \\ California, Los Alamos, NM 87545, U.S.A. \\ (3) Materials Department, University of California, Santa Barbara CA 93106, U.S.A. \\ ( $\left.{ }^{4}\right)$ Theorie II, IFF, KFA Jülich, Postfach 1913, D 5170 Jülich, F.R.G.
}

(Reçu le 8 décembre 1989, accepté le 22 février 1990)

\begin{abstract}
We present a new approach to the linear macroscopic behaviour of polymeric liquids and melts in terms of the macroscopic variables : velocity field, density, entropy density and strain field and we discuss how the classical Maxwell model emerges as a limiting special case. The novel approach used here, which considers the strain field as an additional macroscopic variable that relaxes on the timescale of the Maxwell time, allows to incorporate additional variables such as the macroscopic polarization or the concentration in mixtures easily. We point out a number of new cross-couplings not given before in connection with the Maxwell model and we outline four experiments to test some of the suggestions made. We predict for example that transverse acoustic phonons can be excited in a transient network with a frequency larger than the Maxwell frequency of the polymer melt or solution studied.
\end{abstract}

\section{Introduction and motivation.}

The dynamic behaviour of polymer melts and solutions is known to be complex and complicated, both in the macroscopic (Ref. [1] and Refs. cited therein) and in the microscopic and mesoscopic domain (Refs. [2, 3, 4] and Refs. cited therein). To describe the low frequency, long wavelength behaviour of polymer melts and solutions the most commonly used model is the Maxwell model, which was considered first by Maxwell [5] to "characterize the non-ideal behaviour of gases. To describe the phenomenon that polymer melts and solutions react like a liquid below a certain frequency (usually called the Maxwell frequency) and solid-like above this characteristic frequency, in the classical Maxwell model a dynamic equation for the stress tensor is introduced. This model has been used in the past to study a large number of physical phenomena (Ref. [1] and Refs. cited therein) and more recently for e.g. thermal convection [6-8] and surface waves [9]. To give an accurate description of the experimental results a number of modifications of the classical Maxwell model (e.g. the Jeffreys model) was introduced [1] and the analogy to electric circuits was emphasized and exploited. For none of these models a discussion of the cross-coupling to other macroscopic 
quantities such as temperature or polarization seems to exist. No fundamental reason why there should be a dynamic equation for the stress tensor was ever given to the best of our knowledge.

Here we suggest to use a different gateway of attack to characterize the low frequency, long wavelength behaviour of polymer melts and polymer solutions in the small amplitude limit. Based on the observation that in their liquid state polymers behave like a simple liquid for sufficiently small frequencies and like a solid for frequencies above the Maxwell frequency, we use the hydrodynamic approach (compare for example Refs. [10] and [11]) to describe the macroscopic behaviour of polymer melts and solutions. In writing down hydrodynamic equations one focuses on the long wavelength, low frequency response of a condensed system, which does not have long range forces e.g. due to Coulomb interactions. Then one derives balance equations for the truly conserved quantities such as density and density of linear momentum and for the quantities associated with spontaneously broken continuous symmetries such as the displacement field in crystals [10] or the deviations from the equilibrium direction of the director for nematic liquid crystals [11]. In some cases this rigorous hydrodynamic approach, which keeps only the quantities which do not relax in the limit of infinite wavelength, is modified to keep variables, which relax in a long but finite time, such as for example the modulus of the order parameter close to a phase transition. This approach is called macroscopic dynamics and it has been used first to describe the dynamic behaviour near the $\lambda$-transition in ${ }^{4} \mathrm{He}$ [12] and to many other situations in the following (compare the discussion in Ref. [13]). Recently this concept has been used to study the static behaviour close to the isotropic-nematic transition in liquid crystalline elastomers [14].

In this paper we propose to use the macroscopic dynamic approach to polymers. Apparently this has not been done before. From the observation that polymer melts and solutions behave like a solid or liquid-like depending on the frequency of the perturbation, we conclude immediately that the displacement field, which is a truly hydrodynamic variable in crystals, is the best candidate to serve as a macroscopic dynamic variable in polymer dynamics. Since polymers behave like a liquid for frequencies smaller than the Maxwell frequency, the dynamic equation for the displacement field will acquire a source term, which describes the fact that the displacement acts like a hydrodynamic variable only for frequencies large compared to the Maxwell frequency. As the temperature for the glass transition is approached, the Maxwell time goes to infinity and renders the displacement field a truly hydrodynamic variable as the glassy phase is entered.

In the following it will become clear that the incorporation of this macroscopic variable, the displacement field, will lead to a number of novel cross-coupling effects not considered before in the dynamics of polymeric liquids. In addition, the existence of the displacement field as a macroscopic variable, leads to additional support for the concept of a transient network, which has been put forward in various versions as a microscopic and as mesoscopic concept in many publications (compare Refs. [2] and [4] for a review). In this paper we point out for the first time that one could also use it as a macroscopic concept when viewed as outlined above.

The paper is organized as follows. In section 2 we give the macroscopic dynamic equations and in section 3 we analyze in detail how our approach is connected to the classical Maxwell model. In section 4 we suggest four experiments to test our predictions and in section 5 we briefly summarize the main results and conclude with a perspective. In an Appendix we list the correlation functions of the fluctuating parts of the dissipative currents and discuss the structure they assume in the classical Maxwell model.

\section{The linearized macroscopic equations.}

As already discussed in the introduction we will focus exclusively on linearized macroscopic 
equations throughout the present paper. In the truly hydrodynamic regime, that is for all frequencies $\omega$, which are small compared to all microscopic frequencies $1 / \tau_{\mathrm{c}}: \omega \tau_{\mathrm{c}} \ll 1$ and for all wavevectors $k$ small compared to all wavevectors associated with microscopic length scales $\ell_{\mathrm{c}}: k \ell_{\mathrm{c}} \ll 1$, one has as hydrodynamic variables the densities of all conserved quantities. These are for a simple fluid : the density $\rho$ associated with the law of mass conservation, the density of linear momentum $\mathbf{g}$ (conservation of linear momentum) and the energy density $\varepsilon$ (energy conservation). In addition one has in mixtures without chemical reactions the concentration $c$ as a hydrodynamic variable, since the concentration of one constituent in a mixture cannot be destroyed, but only be redistributed in space.

In the introduction we have already outlined why we want to keep one additional quantity in the list of macroscopic variables, namely the displacement field $\mathbf{u}(\mathbf{r}, t)$. This is not a truly hydrodynamic variable in a polymer melt as the other variables introduced above, since it relaxes in a large, but finite time. Nevertheless we keep the displacement field in our list of macroscopic variables to account for the empirical fact, that polymer melts and solutions behave for small frequencies as a simple liquid, but for sufficiently large frequencies like a solid. On the other hand in a solid the displacement field is a truly hydrodynamic variable, since it does not relax in the long time limit. We also keep in our list of macroscopic variables the density of the macroscopic polarization $\mathbf{P}$, which will mainly be of practical importance for highly polarizable polymer melts. Our goal is now to derive for all these macroscopic variables balance equations valid for long wavelengths and for frequencies smaller than all microscopic frequencies. The Maxwell frequency is considered to be a macroscopic frequency in this context.

The approach used to derive such balance equations is linear irreversible thermodynamics [15]. In the hydrodynamic regime a given system is in thermodynamic equilibrium locally and the validity of the Gibbs relation

$$
T \mathrm{~d} \sigma=\mathrm{d} \varepsilon-\mu \mathrm{d} \rho-Z \mathrm{~d} c-\mathbf{v} \cdot \mathrm{d} \mathbf{g}-\mathbf{E} \cdot \mathrm{d} \mathbf{P}-\Phi_{i j} \mathrm{~d} \varepsilon_{i j}
$$

is guaranteed. This relation can be viewed as a local formulation of the first law of thermodynamics. In contrast to the case of global (that is everywhere in space) thermodynamic equilibrium, the Gibbs relation (Eq. (2.1)) is formulated locally, that is for the vicinity of a fixed, but otherwise arbitrary point in space. This means that - in contrast to global equilibrium - all the quantities in equation (2.1) are fields, which vary spatially and temporally in the hydrodynamic regime. In deriving equation (2.1) one has averaged already over many atoms, or - to phrase it differently - each fluid particle contains enough molecules so that a continuum approximation is justified. Equation (2.1) gives a relation between the increments in the thermodynamic variables. It relates the variation of the entropy density $\sigma(\mathbf{r}, t)$ to the changes of all the other thermodynamic quantities $\rho, \mathbf{g}, \varepsilon, \mathbf{P}$ and $\varepsilon_{i j}$, where $\varepsilon_{i j}$ is the strain tensor, that is the symmetrized gradient of the displacement field : $\varepsilon_{i j}=\frac{1}{2}\left(\nabla_{i} u_{j}+\nabla_{j} u_{i}\right)$. It is the gradient of the displacement field and not the displacement field itself, which enters the Gibbs relation, since homogeneous displacements do not change the energy of the system. Invariance under rotations is guaranteed by the symmetric form of $\varepsilon_{i j}($ curl $\mathbf{u}=0$ ). Nevertheless one must make sure when writing down macroscopic equations in the following that at all steps one only deals with three independent macroscopic quantities, since the displacement field $\mathbf{u}(\mathbf{r}, t)$ has only three components (compatibility condition, $\mathrm{cf}$. equation (2.12) below).

The quantities multiplying the increments in the thermodynamic variables in the Gibbs relation are called thermodynamic conjugate quantities or thermodynamic forces. They express how the energy changes, when one thermodynamic variable is changed while all the 
other ones are kept constant. For example the chemical potential $\mu$ is thus obtained via $\mu=\delta \varepsilon /\left.\delta \rho\right|_{\text {... }}$, where the ellipses indicate that all other thermodynamic variables are kept fixed while the energy is varied with respect to the density. Similarly one obtains the local velocity $\mathrm{v}(\mathbf{r}, t)$ by varying the energy with respect to the density of linear momentum $\mathbf{g}(\mathbf{r}, t)$ or the electric field $\mathbf{E}(\mathbf{r}, t)$ by varying $\varepsilon$ with respect to the polarization density $\mathbf{P}(\mathbf{r}, t)$. The thermodynamic forces in equation (2.1) we have not yet alluded to are the temperature $T(\mathbf{r}, t)$, the relative chemical potential of the mixture $Z(\mathbf{r}, t)$, and the elastic stress $\Phi_{i j}(\mathbf{r}, t)$, the quantity which is the thermodynamic conjugate to the strain field $\varepsilon_{i j}(\mathbf{r}, t)$. Since $\varepsilon_{i j}(\mathbf{r}, t)$ is symmetric, $\Phi_{i j}(\mathbf{r}, t)$ can be chosen to be symmetric without loss of generality.

The static behaviour of the system is now obtained by connecting the thermodynamic forces to the macroscopic thermodynamic variables. As we have seen above these forces can be obtained from the energy, by taking variational derivatives with respect to the variables. For linearized thermodynamics one has two equivalent possibilities to formulate the thermostatic theory : i) either one can expand the thermodynamic forces linearly into the variables or ii) one expands the energy up to second order in the variables. The derivation of the thermodynamic forces from the energy guarantees then automatically that they are linear in the variables. In pursuing either approach, however, the expansion must preserve all fundamental invariance properties of the system under investigation including for example translational and rotational invariance, time reversal symmetry and symmetry under spatial parity.

In the present case of a polymer melt we obtain for the energy $E=\int \mathrm{d} \tau \varepsilon$

$$
\begin{aligned}
E=E_{0}+\int \mathrm{d} \tau\left[\frac{1}{2} c_{i j k \ell} \varepsilon_{i j} \varepsilon_{k \ell}+\varepsilon_{i i}\left(\chi^{\rho} \delta \rho+\chi^{\sigma} \delta \sigma\right.\right. & \left.+\chi^{c} \delta c\right)+ \\
& \left.+\zeta_{i j k \ell} \varepsilon_{i j} \nabla_{k} P_{\ell}+\frac{1}{2} \chi_{i j} P_{i} P_{j}\right]
\end{aligned}
$$

where $E_{0}$ contains all the terms already present in a simple liquid and where the summation convention over repeated indices is implied in equation (2.2) and all the following equations. The tensors $c_{i j k \ell}$ and $\zeta_{i j k \ell}$ take for an isotropic medium generally the form $a_{i j k \ell}=a_{1} \delta_{i j} \delta_{k \ell}+$ $a_{2}\left(\delta_{i k} \delta_{j \ell}+\delta_{i \ell} \delta_{j k}-\frac{2}{3} \delta_{i j} \delta_{k \ell}\right)$, where $c_{1}, \zeta_{1}$ and $c_{2}, \zeta_{2}$ are connected with the trace and the deviator of the corresponding second rank tensors $\left(\varepsilon_{i j}\right.$ and $\left.\nabla_{i} P_{j}\right)$, respectively. Crosscouplings between the trace of the strain tensor and the density, entropy density and concentration variations are expressed by the static susceptibilities $\chi^{\rho}, \chi^{\sigma}$, and $\chi^{c}$, respectively. Note that in equation (2.2) we have to use explicitly the deviations of the variables from their equilibrium values (e.g. $\left.\delta \rho(\mathbf{r}, t)=\rho(\mathrm{r}, t)-\rho_{\mathrm{eq}}\right)$, while under gradients or time derivatives this distinction is unnecessary (since $(\partial / \partial t) \rho_{\mathrm{eq}}=0=\nabla_{i} \rho_{\mathrm{eq}}$ etc.). The tensor $\chi_{i j}\left(=\chi^{\mathrm{p}} \delta_{i j}\right.$ for an isotropic medium) is the electric susceptibility tensor. By inspection of equation (2.2) we see that there is no static cross-coupling between strains and spatially homogeneous electric fields, but only the higher order cross-coupling to field gradients.

As outlined above the forces are then evaluated by taking the variational derivative of the generalized thermodynamic potential with respect to the appropriate variable, while keeping all other variables constant. We find for example for the force $\Phi_{i j}$ :

$$
\Phi_{i j}=\left.\frac{\delta E}{\delta \varepsilon_{i j}}\right|_{\ldots}=c_{i j k \ell} \varepsilon_{k \ell}+\delta_{i j}\left(\chi^{\rho} \delta \rho+\chi^{\sigma} \delta \sigma+\chi^{c} \delta c\right)+\zeta_{i j k \ell} \nabla_{k} P_{\ell} .
$$


For the conservation laws for density, density of linear momentum and concentration we have

$$
\begin{array}{r}
\dot{\rho}+\nabla_{i} g_{i}=0 \\
\dot{g}_{i}+\nabla_{j} \sigma_{i j}=0 \\
\dot{c}+\nabla_{i} j_{i}^{\mathrm{c}}=0
\end{array}
$$

where $\sigma_{i j}$ is the stress tensor and $j_{i}^{\text {c }}$ the concentration current. As we can read off from equations (2.4) and (2.1) the density of linear momentum serves as both, as a variable and as the current of the density. The balance equations for the non-conserved fields read

$$
\begin{aligned}
\dot{\sigma}+\nabla_{i} j_{i}^{\sigma} & =\frac{R}{T} \\
\dot{\varepsilon}_{i j}+X_{i j} & =0 \\
\dot{P}_{i}+j_{i}^{\mathrm{P}} & =0 .
\end{aligned}
$$

In equations (2.5) $j_{i}^{\sigma}$ is the entropy current, $j_{i}^{\mathrm{P}}$ the polarization current, $X_{i j}$ the quasi-current associated with the strain tensor and the source term $R / T$ in the dynamic equation for the entropy density is the entropy production. The second law of thermodynamics reads $R \geq 0$ for dissipative and reversible processes, respectively. To guarantee rotational invariance of the dynamic equation for the strain field, its quasi-current $X_{i j}$ must be symmetric $X_{i j}=X_{j i}$. From equations (2.4) and (2.5) the conservation law for the energy density follows by making use of the Gibbs relation (2.1). Having written down already the dynamic equations for all the other dynamic variables, the form of the energy current is thus fixed completely. To close the set of macroscopic equations it is now necessary to connect the currents and quasi-currents $g_{i}, \sigma_{i j}, j_{i}^{\sigma}, j_{i}^{\mathrm{c}}, j_{i}^{\mathrm{P}}$ and $X_{i j}$ to the thermodynamic conjugate quantities $T, \mu, Z, \mathbf{v}, \mathbf{E}$ and $\Phi_{i j}$ taking into account all symmetry properties of the system. This step contains as a simple special case for example Fourier's law, which connects the heat current $j_{i}^{\sigma}$ to the appropriate thermodynamic force, the temperature $T$ via

$$
j_{i}^{\sigma}=\kappa \nabla_{i} T
$$

for an isotropic system with thermal diffusivity $\kappa$. In the present paper we concentrate on the linear connection between forces and fluxes, since we are focusing on the linearized macroscopic dynamics.

All the currents written down in equations (2.4) and (2.5) can be split into dissipative and into reversible parts, depending on whether they give rise to a finite amount of dissipation (or positive entropy production) - such as for example the heat current (2.6) - or to a vanishing entropy production $(R=0)$.

Using general symmetry and invariance arguments we obtain for the linear, reversible parts of the currents

$$
\begin{aligned}
g_{i}^{R} & =\rho_{0} v_{i} \\
\sigma_{i j}^{R} & =p \delta_{i j}-\Phi_{i j} \\
j_{i}^{c R} & =c_{0} v_{i} \\
j_{i}^{\sigma R} & =\sigma_{0} v_{i} \\
j_{i}^{P R} & =0 \\
X_{i j}^{R} & =-A_{i j}
\end{aligned}
$$


where $p$ is the hydrostatic pressure and where $A_{i j}$ is the symmetric velocity gradient $A_{i j}=\frac{1}{2}\left(\nabla_{i} v_{j}+\nabla_{j} v_{i}\right)$. The reversible current for the strain field reflects the fact that for homogeneous translations (solid body translations) $\dot{\mathbf{u}}=\mathbf{v}$. The term $\Phi_{i j}$ in the stress tensor is the elastic contribution from the transient network. The quantities $\rho_{0}, c_{0}$ and $\sigma_{0}$ are the equilibrium values of density, concentration and entropy density around which the linearized equations are derived. All the reversible currents given in equation (2.7) lead to vanishing entropy production : $R=0$.

For the derivation of the dissipative parts of the currents in equation (2.6) one has two equivalent possibilities : i) either one expands the currents up to linear order in the forces, or ii) one expands the dissipation function to second order in the thermodynamic conjugates and then obtains the dissipative currents by taking the variational derivatives of the dissipation function with respect to the forces.

We find for the dissipation function

$$
\begin{aligned}
R=R_{0}+\int \mathrm{d} \tau\left[\frac{1}{4 \tau} \Phi_{i j}^{2}+\frac{1}{2} \gamma\left(\nabla_{j} \Phi_{i j}\right)^{2}+\frac{1}{2} \sigma^{E} E^{2}+\right. \\
\left.\quad+\left(\nabla_{j} \Phi_{i j}\right)\left(\zeta^{T} \nabla_{i} T+\zeta^{c} \nabla_{i} Z+\zeta^{E} E_{i}\right)\right]
\end{aligned}
$$

where $R_{0}$ is the part of the dissipation function already present in simple fluids. All the terms in equation (2.8) have been chosen in such a way that they guarantee the positivity of the entropy production in accordance with the second law of thermodynamics, if the phenomenological transport parameters satisfy a number of positivity conditions including $\tau>0$, $\gamma>0, \sigma^{E}>0, \gamma \sigma^{E}>\left(\zeta^{E}\right)^{2}$. In addition all contributions to $R$ transform like a scalar, that is they must be invariant under translations, rotations, under time reversal and under operations including spatial parity.

The quantity $\tau$ is the relaxation and $\gamma$ the selfdiffusion constant of the strain field and $\sigma^{E}$ is the electric conductivity. Inspecting equation (2.8) we see that there are several dissipative cross-couplings between the strains in the transient network on one hand and between the gradients of temperature and concentration and of an electric field on the other. It seems worthwhile to notice that none of these terms has been considered in the classical version of the Maxwell model. Some experimentally testable consequences of these crosscoupling terms will be highlighted in section 4 .

As already mentioned above we get the dissipative parts of the currents by taking the variational derivative of the dissipation function with respect to the appropriate thermodynamic force. We find for example for the dissipative current associated with the dynamic equation for the strain tensor

$$
X_{i j}^{\mathrm{D}}=\left.\frac{\delta R}{\delta \Phi_{i j}}\right|_{\ldots}=\frac{\Phi_{i j}}{2 \tau}-\nabla_{j}\left(\gamma \nabla_{k} \Phi_{i k}+\zeta^{T} \nabla_{i} T+\zeta^{c} \nabla_{i} Z+\zeta^{E} E_{i}\right)
$$

From equation (2.9) we read off immediately that it can be split into a source term and into a part which can be expressed as the gradient of a vector :

$$
X_{i j}^{D}=\frac{\Phi_{i j}}{2 \tau}-\tilde{X}_{i j}
$$

with

$$
\tilde{X}_{i j}=\frac{\gamma}{2}\left(\nabla_{i} \nabla_{k} \Phi_{j k}+\nabla_{j} \nabla_{k} \Phi_{i k}\right)+\nabla_{j} \nabla_{i}\left(\zeta^{T} T+\zeta^{c} Z\right)+\frac{\zeta^{E}}{2}\left(\nabla_{i} E_{j}+\nabla_{j} E_{i}\right)
$$


where we have written $\tilde{X}_{i j}$ in an explicitly symmetric form and where we have made use of the relation curl $\mathbf{E}=\mathbf{0}$ of electrostatics. The requirement that the dynamic equation for the strain tensor transforms the same way as the strain tensor itself implies a restriction. From classical elasticity theory one knows that the strain tensor must satisfy a compatibility condition to guarantee that a continuous displacement field $\mathbf{u}(\mathbf{r}, t)$ exists. This condition reads

$$
\varepsilon_{i j k} \varepsilon_{\ell m n} \nabla_{j} \nabla_{m} \varepsilon_{k n}=0
$$

and applies, because of equation (2.5), also to $X_{i j}\left(\varepsilon_{i j k} \varepsilon_{\ell_{m n}} \nabla_{j} \nabla_{m} X_{k n}=0\right)$. Obviously, the reversible part $X_{i j}^{R}$ and $\tilde{X}_{i j}$ fulfill this condition automatically, but not the source term $\Phi_{i j} /(2 \tau)$ in equation (2.10). The latter obeys $\varepsilon_{i j k} \varepsilon_{\ell_{m n}} \nabla_{j} \nabla_{m} \Phi_{k n}=0$ only if the elastic moduli (cf. Eq. (2.2)) satisfy the condition

$$
c_{2}=\frac{3}{2} c_{1}
$$

that is bulk and shear modulus of a polymer melt above the Maxwell frequency are not independent, but satisfy within a linearized macroscopic dynamic theory equation (2.13). Condition (2.13) guarantees that the relaxation times for longitudinal strains and for transverse strains, $3 c_{1} /(2 \tau)$ and $c_{2} / \tau$, respectively, are equal. For a true elastic solid, there is no counterpart to condition (2.13) (there is no source term in $X_{i j}^{\mathrm{D}}$, if there is no relaxation) and the two elastic moduli are independent. On the other hand we have kept the compressibility (i.e. the density dependence of the pressure in Eq. (2.7)), so that in our description of polymer melts the response to bulk and shear stresses is still given by two independent quantities.

\section{Comparison of the present approach with the Maxwell model in its classical formulation.}

In its classical formulation the Maxwell model takes the following form using a dynamic equation for the off-diagonal part of the stress tensor, $\sigma_{i j}^{\prime}$

$$
\left(t_{0} \frac{\partial}{\partial t}+1\right) \sigma_{i j}^{\prime}=-2 \nu A_{i j}
$$

with Maxwell time $t_{0}$ and shear viscosity $\nu$. From the point of view of the derivation of macroscopic equations it is not clear why the equation for the stress tensor should be dynamic. From equation (3.1) and the momentum conservation equation it is also not clear how to get couplings to other macroscopic variables including entropy density, concentration in mixtures and macroscopic polarization.

To elucidate the similarities and differences between the two approaches in detail we take a Fourier transform of equation (3.1) which yields

$$
\sigma_{i j}^{\prime}=-2 \nu A_{i j}\left(1+i \omega t_{0}\right)^{-1} \text {. }
$$

Next we combine this equation with the linearized momentum conservation equation

$$
\rho_{0} \frac{\partial}{\partial t} v_{i}+\nabla_{j} \sigma_{i j}=0
$$

and obtain after Fourier transformations in time and space (for incompressible liquids)

$$
\rho_{0} \omega v_{i}+k_{i} p-i k^{2} v_{i} \frac{\nu}{1+i \omega t_{0}}=0
$$


It is this equation (3.4), which can be compared most easily with the approach put forward in the present paper. To see this we start from the suitably reduced equation (2.5) (using Eqs. (2.7) and (2.9))

$$
\frac{\partial}{\partial t} \varepsilon_{i j}-A_{i j}-\frac{\gamma}{2}\left(\nabla_{j} \nabla_{k} \Phi_{i k}+\nabla_{i} \nabla_{k} \Phi_{j k}\right)=-\frac{1}{2 \tau} \Phi_{i j}
$$

where we have discarded as discussed above all couplings to the other macroscopic variables and assumed incompressibility in order to be able to compare with the classical Maxwell model. From the (generalized) Navier-Stokes equation (cf. (2.4) and (2.7))

$$
\rho_{0} \frac{\partial}{\partial t} v_{i}+\nabla_{i} p-\nabla_{j} \Phi_{i j}=\eta_{2} \Delta v_{i}
$$

with the help of equation (2.2) and after taking Fourier transforms, equations (3.5) and (3.6) combine to

$$
\rho_{0} \omega v_{i}+k_{i} p-i k^{2} v_{i}\left\{\eta_{2}+\frac{\tau}{1+i \omega \tau / c_{2}+\gamma \tau k^{2}}\right\}=0
$$

Equations (3.4) and (3.7) are very convenient for the comparison of the predictions of the Maxwell model and of the macroscopic dynamics approach. Looking at the coefficient of $v_{i} k^{2}$ we see that $\nu\left(1+i \omega t_{0}\right)^{-1}$ is replaced by $\eta_{2}+\tau\left(1+i \omega \tau / c_{2}+\gamma k^{2} \tau\right)^{-1}$ using the present macroscopic dynamics approach.

To carry out the comparison explicitly it turns out to be very efficient to investigate various limiting cases. For the low frequency regime $(\omega \rightarrow 0)$ we obtain $\nu$ for the classical Maxwell model and $\eta_{2}+\tau\left(1+\gamma k^{2} \tau\right)^{-1}$ in the present framework. We read off immediately that in the long wavelength limit the appropriate expression is $\eta_{2}+\tau$ and $\nu$ in the classical model. Neglecting the dispersion $\left(\gamma k^{2} \tau\right)$ is justified as long as it is small compared to 1. Maximal absorption is obtained for both, $\omega t_{0}$ and $\omega \tau / c_{2}$ of order one for the two approaches compared here, which is in agreement with one's intuition. Both approaches also agree in the limit of vanishing Maxwell time, in which they give rise to the behaviour of a simple liquid. For the classical Maxwell model the dynamic equation for the stress tensor reduces to the constitutive law of a simple incompressible liquid and in the case of the macroscopic dynamic approach the strain tensor no longer acts as a macroscopic (slow) variable in the $\tau \rightarrow 0$ limit.

By construction the macroscopic dynamics approach gives the correct limit for the case of an infinite Maxwell time. In this case the hydrodynamic equations for a solid result as it must be the case. For the classical Maxwell model it is quite complicated to obtain this limit, since the classical model depends only on $\omega t_{0}$ thus making it impossible to investigate the limit of a solid, since the macroscopic behaviour can only be expected to be obtained correctly for sufficiently small frequencies. Otherwise microscopic modes are being excited. In the macroscopic dynamics approach $\omega$ and $\tau$ are independent quantities and it is therefore staightforward to take the $\tau \rightarrow \infty$ limit.

From the point of view of macroscopic dynamics there is no justification for models which incorporate a whole sequence of microscopic times. In the present gateway of attack only the Maxwell time is incorporated. All shorter time scales are considered to be truly microscopic. However, it is conceivable that there exist additional long time scales originating from microscopic or mesoscopic processes. In that case, additional macroscopic variables must be kept. We note that the present approach loses its appealing conceptual simplicity if there are too many of these additional variables. 


\section{Possible experimental tests of the macroscopic dynamics approach.}

Naturally it seems very important to test the concept put forward above experimentally. In this section we outline four simple, but nontrivial possibilities.

As we have seen in the last section, the additional degrees of freedom can be excited at sufficiently high frequencies by applying for example extensional flow or simple shear. This occurs predominantly through the direct reversible coupling between temporal strain variations and velocity gradients. Having generated these strains in the transient network they are dissipated on the time scale of the Maxwell time. This dissipation leads via the corresponding term in the entropy production to a heating effect on the sample to quadratic order in the amplitude of the applied field

$$
\dot{T} \sim \int \mathrm{d} V \frac{1}{\tau} \varepsilon_{i j}^{2} .
$$

Thus one can detect by a dc measurement (observation of the mean sample temperature as a function of time) the consequences of the presence of the transient network. For sufficiently small shearing rates with frequencies smaller than the Maxwell frequency only the ordinary viscous heating present in all simple fluids arises whereas for higher frequencies additional dissipation channels are being opened due to the presence of the transient network. To make sure that the additional heating observed is due to the mechanism presented here and not due to higher order nonlinearities one can plot for fixed frequency the temperature increase as a function of shearing amplitude. It is then the quadratic part of the small amplitude response which is of interest here. If one plots the temperature increase as a function of frequency, one should observe a change in the value of the slope at a frequency comparable to the Maxwell frequency. There are indications from experiments done in engineering [16] that the effect described here might be at least partially responsible for the drastic heating effects observed in polymer melts under high shear. Since the reported heating effects are rather dramatic (up to $1000 \mathrm{~K} / \mathrm{s}$ ) it can be expected that chemical reactions and even decomposition take place at those elevated temperatures thus changing significantly the physical properties of the melt. This would explain why a second experiment carried out on the same sample leads to strong hysteresis effects and to a completely different physical response.

Looking at the approach taken here which closely parallels that used to derive hydrodynamic equations for a solid, another possibility to test the concept of a transient network comes into mind immediately. To do this one investigates the behaviour of a polymeric sample in a stationary sound resonator. Depending on whether the frequency used for excitation is above or below the Maxwell frequency, a transient network and thus a finite shear modulus is probed or not $[1,17]$. To test whether this picture makes sense one can now excite transverse acoustic phonons. We predict that this is possible only for frequencies above the Maxwell frequency. The detection of such propagating transverse acoustic phonons, which do not exist in a simple fluid, would yield the perhaps most convincing evidence for the validity of the picture of a transient network. For frequencies below the Maxwell frequency such a propagative transverse mode could not be observed and one should rather see the purely dissipative vorticity diffusion, which is well known from simple liquids. To the best of our knowledge an experiment to excite transverse sound waves has not been done, but it should be fairly straightforward to fill this gap. We should also like to point out that only the picture given here prompts one to perform such an experiment, since it is not at all obvious how to interpret the outcome of such a measurement using the classical Maxwell model.

For highly polarizable polymer melts another possibility arises. Inspecting the structure of the dissipation function (Eq. (2.8)), we see that it contains e.g. a term $\zeta^{E} E_{i} \nabla_{j} \Phi_{i j}$. Taking a 
spatial and temporal Fourier transform of the resulting equation for the strain, we obtain the following equation

$$
i \omega \varepsilon_{i j}=-\frac{\Phi_{i j}}{2 \tau}+\frac{i}{2} \zeta^{E}\left(k_{i} E_{j}+k_{j} E_{i}\right)+\cdots
$$

where the ellipses indicate the terms irrelevant for the argument given here. Thus we read off from equation (4.2) that a spatially inhomogeneous ac electric field leads to a strain in the transient network which in turn leads to heating effects. We stress that a spatially homogeneous ac electric field is not sufficient, since it does not couple to strains to quadratic order in the macroscopic variables. Nevertheless a set-up with a spatially homogeneous electric field will be useful to evaluate the effects due to Ohmic heating which are always present. A highly polarizable polymer is mainly needed to carry out the experiment suggested here because otherwise the effect predicted might be masked completely by the trivial effect of Ohmic heating. In a sense this experiment using ac spatially inhomogeneous electric fields is somewhat complementary to the case of mechanical shear in that one now has a different external driving field, namely an electric field gradient instead of a velocity gradient. Below the Maxwell frequency one should again observe only Ohmic heating even in a spatially inhomogeneous electric field.

For polymer melts with a very large Maxwell time, that is for systems which behave very much like a solid already, an even more direct test for the concept of a transient network seems feasible experimentally. In this case one could investigate the linear coupling between velocity gradients, strains and temperature variations directly for sufficiently small frequencies above the Maxwell frequency. One would have to measure the temperature variations in a time-resolved experiment and one should observe ac temperature variations which are proportional to the amplitude of the applied shear for small shear rates, an effect which cannot possibly exist in a simple liquid or in a polymer below the Maxwell frequency. Also for this experiment we are not aware of any published experimental results nor of any motivation to carry out such an experiment based on the formulation of the classical Maxwell model. This is even more the case for the coupling to the electric field gradients. We close this section by pointing out that a magnetic field cannot induce an effect analogous to the electric field due to the difference of the behaviour under time reversal of $\mathbf{E}$ and $\mathbf{B}$.

\section{Conclusions and perspective.}

In this paper we have analyzed within the framework of macroscopic dynamics the long wavelength, low frequency behaviour of polymer melts and polymer solutions in the limit of small amplitude motion. We have discussed in detail the relation of the classical Maxwell model to our approach and we have suggested four experiments, which could test the validity and clarify the limitations of our approach and yield clear-cut evidence for the picture of a transient network. It turns out that there is no physical motivation to write down a dynamic equation for the stress tensor as it is even being done in the physics textbook literature [18]. It also emerges that the use of the displacement field as the macroscopic variable reveals very easily cross-couplings to the other macroscopic variables such as temperature and macroscopic polarisation, which have not been previously considered. From the dynamic equation for the strain we can read off in our approach immediately that the melt behaves like a solid for large frequencies and like a liquid for frequencies small compared to the Maxwell frequency. A resort to various modifications of electric circuit models is therefore unnecessary using the present gateway of attack leaving alone that those circuit type models cannot incorporate thermomechanical and electromechanical effects which have been shown to be very important for the characterization of the macroscopic behaviour in this paper. 
The biggest challenge at this time seems to be to get reliable quantitative estimates for the novel cross-couplings. We note, however, that even for classical elastomers no quantitative calculations for these coefficients appear to be available [19]. We feel, however, that this task might be easier in some respect for the transient network, since it is spatially homogeneous and thus not suffering from problems such as inhomogeneous cross-linking densities, etc.

Having laid the foundation of a macroscopic dynamic description of polymer melts and solutions in this paper, we will present its generalizations in the near future.

\section{Acknowledgements.}

H.R.B. and H.P. thank the Deutsche Forschungsgemeinschaft for support. The work done at the Center for Nonlinear Studies, Los Alamos National Laboratory has been performed under the auspices of the United States Department of Energy.

\section{Appendix.}

In this appendix we derive the formulas for the correlation functions within fluctuating hydrodynamics [20]. From statistical mechanics it is well known that dissipation is necessarily accompanied by fluctuations. Having vanishing mean values these fluctuations can be neglected in a deterministic treatment, but for various applications of linear hydrodynamic equations it is fruitful to allow for these fluctuating parts in the dissipative currents. In equilibrium (or near it) the correlation functions of the fluctuating parts of the currents are related to the dissipative transport parameters by the fluctuation-dissipation-theorem. Denoting by $J_{i}^{\sigma}, \Sigma_{i j}, J_{i}^{c}, \xi_{i j}$, and $J_{i}^{P}$ the fluctuating parts of the currents $j_{i}^{\sigma}, \sigma_{i j}$, $j_{i}^{c}, X_{i j}$, and $j_{i}^{P}$ respectively, and assuming that they are described by Gaussian, white noise stochastic processes, standard textbook procedures [20] lead to the correlation functions

$$
\left\langle\xi_{i j}(\mathbf{r}, t) \xi_{k \ell}\left(\mathbf{r}^{\prime}, t^{\prime}\right)\right\rangle=k_{\mathrm{B}} T(2 \tau)^{-1} D_{i j k \ell} \delta\left(\mathbf{r}-\mathbf{r}^{\prime}\right) \delta\left(t-t^{\prime}\right)
$$

and

$$
\left\langle\nabla_{j} \xi_{i j}(\mathbf{r}, t) J_{k}^{\alpha}\left(\mathbf{r}^{\prime}, t^{\prime}\right)\right\rangle=2 k_{\mathrm{B}} T \zeta^{\beta} \delta_{i k} \delta\left(\mathbf{r}-\mathbf{r}^{\prime}\right) \delta\left(t-t^{\prime}\right)
$$

where $k_{\mathrm{B}}$ is Boltzmann's constant, $D_{i j k \ell}=\delta_{i k} \delta_{j \ell}+\delta_{i \ell} \delta_{j k}$ and where we have neglected the diffusion-like $\gamma$-contribution against the relaxation $(\sim 1 / \tau)$ of the transient network. Equation (A.2) applies for the pairs $(\alpha, \beta)=(\sigma, T),(c, c)$, and $(P, E)$ only. All other correlation functions are as in simple liquids, e.g.

$$
\left\langle\Sigma_{i j}(\mathbf{r}, t) \Sigma_{k \ell}\left(\mathbf{r}^{\prime}, t^{\prime}\right)\right\rangle=2 k_{\mathrm{B}} T \eta_{i j k \ell} \delta\left(\mathbf{r}-\mathbf{r}^{\prime}\right) \delta\left(t-t^{\prime}\right)
$$

where $\eta_{i j k \ell}$ is the (isotropic) viscosity tensor having the same tensorial structure as $a_{i j k \ell}$, defined after equation (2.2). From equations (A.1)-(A.3) it is straightforward to derive the correlation functions of all hydrodynamic variables desired in a given situation.

In order to compare with the classical Maxwell model, we will eliminate the elastic part in the stress tensor with the help of equation (2.7). After spatial and temporal Fourier transformation we are left with an effective stress tensor, whose fluctuating part, $S_{i j}$, is then a linear combination of $\Sigma_{i j}$ and $\xi_{i j}$

$$
S_{i j}(k, \omega)=\Sigma_{i j}(k, \omega)+\left(i \omega \underline{\mathbf{c}}^{-1}+(2 \tau)^{-1} \underline{\mathbf{D}}\right)_{i j k \ell}^{-1} \xi_{i j}(k, \omega)
$$


where $\underline{c}^{-1}$ is the inverse of the elasticity tensor (cf. (2.2)). For the correlation function of this effective fluctuating stress tensor we find

$$
\begin{array}{rl}
\left\langle S_{i j}(k, \omega) S_{k \ell}\left(k^{\prime}, \omega^{\prime}\right)\right\rangle=2 k_{\mathrm{B}} & T \delta\left(k-k^{\prime}\right) \delta\left(\omega-\omega^{\prime}\right) \times \\
\times & {\left[\left(\eta_{2}+\frac{\tau}{1+\omega^{2}}\right) D_{i j k \ell}+\left(\eta_{1}-\frac{2}{3} \eta_{2}\right) \delta_{i j} \delta_{k \ell}\right]}
\end{array}
$$

with $\hat{\omega}=\omega \tau / c_{2}$. Equation (A.5) is the appropriate formula to be compared with the classical Maxwell model.

\section{References}

[1] Ferry J. D., Viscoelastic Properties of Polymers (Wiley, N. Y., 2nd Edition) 1970.

[2] Graessley W. W., Adv. Polym. Sci. 16 (1974) 1.

[3] DE Gennes P. G., Scaling Concepts in Polymers (Cornell University Press : Ithaca, N. Y.) 1979.

[4] Dor M., Edwards S. F., The Theory of Polymer Dynamics (Clarendon Press : Oxford) 1986.

[5] MaXwell J. C., Phil. Trans. R. Soc. A 157 (1867) 49.

[6] Vest C. M., ARPaCCI V. S., J. Fluid Mech. 36 (1969) 613.

[7] Brand H. R., Zielinska B. J. A., Phys. Rev. Lett. 57 (1986) 3167.

[8] Zielinska B. J. A., Mukamel D., Steinberg V., Phys. Rev. A 33 (1986) 1454.

[9] Pleiner H., Harden J. L., Pincus P. A., Europhys. Lett. 7 (1988) 383 and Harden J. L., Pleiner H., Pincus P. A., Langmuir 5 (1989) 1436.

[10] Martin P. C., Parodi O., Pershan P. S., Phys. Rev. A 6 (19.72) 2401.

[11] FORSTER D., Hydrodynamics, Broken Symmetry and Correlation Functions (Benjamin, Reading, Mass) 1975.

[12] Khalatnikov I. M., Introduction to the Theory of Superfluidity (Benjamin, Reading, Mass) 1965.

[13] Pleiner H., Incommensurate Crystals, Liquid Crystals and Quasicrystals, J. F. Scott and N. A. Clark Eds. (Plenum, New York) 1987.

[14] Brand H. R., Makromol. Chem. Rap. Comm. 10 (1989) 67 and 441.

[15] DE Groot S. R., MAZur P., Nonequilibrium Thermodynamics (North-Holland : Amsterdam) 1962.

[16] Gleissle W., Ph. D. Thesis, Universität Karlsruhe, West Germany (1978).

[17] De Gennes P. G., Pincus P. A., J. Chimie Phys. 74 (1977) 616.

[18] Landau L. D., Lifshitz E. M., Elasticity Theory (Pergamon Press, New York) 1959.

[19] Treloar C. R. G., Rep. Progr. Phys. 36 (1973) 755.

[20] Landau L. D., Lifshitz E. M., Hydrodynamics (Pergamon Press, New York) 1959. 